\title{
Nuevas métricas de audiencia al servicio del Inbound Marketing
}

\section{New Audience Metrics at the Service of Inbound Marketing}

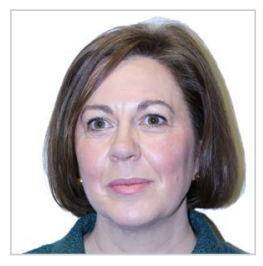

Guadalupe Aguado-Guadalupe. Profesora Titular del Departamento de Periodismo y Comunicación Audiovisual de la Universidad Carlos III. Doctora en Ciencias de la Información por la Universidad Complutense de Madrid. Miembro del grupo de investigación PASEET. Su línea de investigación son los modelos de negocio de las empresas informativas en el entorno digital. Ha publicado en revistas de Estados Unidos, Australia, España, Portugal, México, Brasil, Colombia, Ecuador y Perú. Su última publicación es "Repercusión de las métricas de audiencia online en la comercialización publicitaria del producto informativo”, en Razón y Palabra, 2017.

Universidad Carlos III de Madrid, España

maguado@hum.uc3m.es

ORCID: 0000-0001-7314-2403

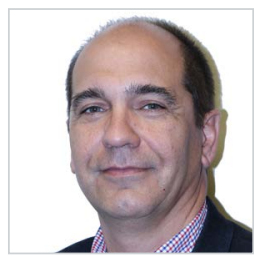

Alberto Luis García-García. Profesor Contratado Doctor en la Facultad de Ciencias de la Información de la Universidad Complutense de Madrid. Experto en Nuevas Tecnologías e Industrias Culturales. Sus líneas de investigación se centran en la relación entre la tecnología y nuevas técnicas de producción en relación a la propuesta de nuevos modelos de negocio en el ámbito de la comunicación digital. Entre sus últimos artículos publicados se encuentra "Publicidad en la era de la postproducción: El producto virtualizado" en la revista Pensar la publicidad". Universidad Complutense de Madrid, España algarci@ucm.es

ORCID: 0000-0002-6805-6700

\section{Resumen:}

En el presente artículo se aborda la utilidad de las herramientas y métricas de audiencia surgidas en el entorno digital para desarrollar prácticas comunicativas de Inbound Marketing. Los objetivos que se persiguen son reconocer qué nuevos indicadores están utilizando las métricas de audiencia; identificar los datos que aportan para prácticas de inbound marketing y apreciar en qué permiten testar los contenidos y planificarlos. Para ello se identifican las principales herramientas de medición y los servicios que ofrecen, así como las métricas que permiten detectar los comportamientos y emociones de los usuarios frente a los mensajes, posibilitando testar los mismos y planificarlos para atraer la atención del público, retenerlo y fidelizarlo. Las conclusiones permiten ver cómo las nuevas métricas están reconfigurando la manera en la que se producen y distribuyen los contenidos, hasta llegar a convertirse en herramientas

\begin{abstract}
:
This article addresses the usefulness of audience metrics and the tools for developing communicative practices of Inbound Marketing in the digital environment. The objectives of this article are to identify what new indicators audience metrics are using, determine the data they provide for inbound marketing practices, assess how data allows contents to be tested and plan them. For this purpose, the central measurement tools were identified and the services they offer as well as the metrics that allow user behavior and emotions regarding messages to be detected, enabling content testing and planning accordingly to attract and retain audience attention and foster loyalty. The conclusions reveal how new metrics are reshaping the way in which contents are produced and distributed, making them indispensable tools for identifying the capacity to attract, retain, satisfy and create loyal users. Data analysis has become a tool
\end{abstract}

\section{Cómo citar este artículo:}

Aguado-Guadalupe, G. y García-García, A. L. (2018). Nuevas métricas de audiencia al servicio del Inboud Marketing. Doxa Comunicación, 26, 81-98. 
indispensables, que permiten identificar la capacidad de atracción, retención, fidelización y satisfacción de los usuarios frente a dichos contenidos. La analítica de datos ha pasado a ser una herramienta en la que los aspectos pragmáticos interseccionan con aspectos emocionales, permitiendo cuantificar la experiencia y la emoción más allá del hábito y posibilitando planificar los contenidos para adaptarlos a los requerimientos de los usuarios.

Palabras clave:

Inbound marketing; audiencia; métrica de comportamiento; análisis de sentimiento; neuromarketing in which practical aspects intersect with emotional ones, allowing the experience and the emotion beyond habit to be quantified, as well as enabling contents to be planned, adapting them to users' requirements.

\section{Keywords:}

Inbound marketing; audience; behavior metrics, sentiment analysis; neuromarketing.

\section{Introducción}

En la conferencia IDC Predictions 2017, celebrada el 31 de enero de 2017, Marta Muñoz, Resarch \& Operations Director de IDC, resaltó que en 2019, el 40\% de las organizaciones creará nuevos servicios y líneas de negocio para monetizar el dato y que en 2020 el 45\% de las infraestructuras y estructura de negocio de software estará alojado en la nube, además más del 50\% de las corporaciones tendrán proyectos de desarrollo para la movilidad y, de esta manera, obtener ventajas competitivas respecto a la competencia. En dicho contexto, los datos son necesarios en todo tiempo y lugar con el fin de que las empresas puedan interpretarlos y convertirlos en información.

Toda esta gestión se realiza a través de indicadores de rendimientos vinculados a objetivos (KPIs), que giran en relación a conceptos como el retorno de la inversión, en cuyo cálculo son esenciales las métricas que faciliten información sobre interacciones, engagement, conversiones, fidelización y sentimientos. Variables todas ellas que han adquirido especial relevancia en el conocimiento previo y a posteriori del comportamiento de los públicos, en tanto que ello permite generar contenidos acorde a lo requerido por las prácticas comunicativas como el inbound marketing (Halligan y Shah, 2009), que han logrado protagonismo en el entorno digital y con las "que se pretende llegar al consumidor de una manera no intrusiva en la red, descartando acciones que molesten al usuario y provocan la irrupción indeseada de su actividad" (Castelló-Martínez, 2013: 1).

Se puede decir que el inbound marketing se compone de tres elementos: 1) contenidos de interés; 2) estrategias de marketing para atraer tráfico a las páginas y 3) capacidad de educar al usuario para que sea receptivo a la oferta. Así pues, para comprender la importancia de las métricas de audiencia y herramientas de medición surgidas en el entorno digital para el desarrollo de estrategias de inbound marketing hay que partir de que el inbound marketing no sólo se orienta a conseguir clientes finales, sino también a mantenerlos satisfechos y cuidar los prescriptores (Aguado-Guadalupe, 2015). Su finalidad no sólo es atraer clientes, sino convertirlos y finalmente fidelizarlos. Para ello se asienta en tres pilares: la personalización, el contenido y la distribución. De manera que no sólo es relevante la creación de contenidos sino también su distribución de manera pertinente, sin llegar a resultar intrusivo para el público destinatario.

Para llevar a término el desarrollo de estrategias de inbound marketing se requiere tanto un detallado conocimiento de los públicos como la combinación de diversas técnicas de marketing que permiten generar confianza. De manera que se 
hace necesario combinar técnicas de marketing de contenido, usando el contenido para atraer la atención y participación de los usuarios (Sanagustín, 2013); de marketing de permiso, lo que implica la autorización expresa del cliente (Godin, 2001); de marketing relacional, buscando mantener una relación estable en el tiempo, siempre y cuando ambas partes obtengan valor por el mantenimiento de la misma (Barroso-Castro y Marín-Armario, 1999); y de marketing de influencia, término que comenzó a utilizarse en los años 60 por Daniel Edelman, fundador de la multinacional de relaciones públicas Edelman, para denominar el poder que ejercían los famosos sobre los consumidores. Poder que en entorno digital ha ido abriendo paso a otros sectores.

En esta línea han de tenerse presentes las palabras de Sanagustín (2013) cuando explica que el marketing de contenidos es impulsor de la conversión digital, en tanto que cuanto más identificado se encuentre el público con el contenido mayor será la capacidad de engagement. Precisamente el valor que ha adquirido la implicación que se genera entre el público y los contenidos ha llevado a que, como resalta Marshall (2004), el usuario haya adquirido una dimensión interactiva, que hay que tener especialmente en cuenta. Al tiempo que, como señala Del Pino (2009: 8): "la comunicación deviene en un contexto en el que la vinculación emocional entre usuario y marca se convierte en el objetivo del anunciante".

Así pues, se ha ido dando paso a un entorno en el que imperan nuevas prácticas comunicativas que para llevarse a término requieren a su vez de nuevos sistemas de métricas. De manera que se ha dado lugar no sólo a las denominadas métricas de sentimientos, sino también a métricas basadas en la combinación de algoritmos, que como indican Martínez y Lara (2014: 579) permiten el análisis de tendencias y facilitan "estrategias selectivas de contenidos, valorar modelos económicos de la red, gestionar perfiles que consigan configurar amplias redes de usuarios y mejorar el retorno de la inversión en social media". En este sentido, Lamas (2010: 96) señala que la misma complejidad tecnológica digital "es la que introduce nuevas y más precisas posibilidades de seguimiento".

En el presente trabajo se parte de la hipótesis de que la aparición de nuevas métricas en el entorno digital ha facilitado un mayor acercamiento al conocimiento de los comportamientos del público con respecto a los contenidos y a los sentimientos que los mismos provocan, así como a un conocimiento previo que permite la planificación de los contenidos previamente a su difusión. Factores todos ellos que contribuyen al desarrollo de estrategias de inbound marketingy especialmente de marketing de atracción y de marketing de contenidos.

Ello ha llevado a plantear como objeto de estudio del presente artículo el análisis de la importancia y trascendencia que suponen dichas nuevas métricas para el desarrollo de estrategias comunicativas, y especialmente en el diseño y distribución de contenidos de interés que capten la atención de los usuarios.

Para ello se han establecido tres objetivos:

1. Reconocer qué nuevos indicadores se están utilizando en las métricas de audiencia.

2. Identificar los datos que aportan las métricas para prácticas de inbound marketing, especialmente en el caso del marketing de contenidos.

3. Apreciar en qué medida dichas métricas permiten testar los contenidos y planificarlos. 
Para realizar el estudio se ha partido de las aportaciones previas sobre medición de audiencia que han venido abordando el tema desde diferentes perspectivas. Destacable, en dicho sentido es la aportación de Maldonado (2015), quien profundiza en la analítica web; o de Medinaveitia y Merchante (2015) que abordan las dificultades y desafíos que comporta el entorno digital a este respecto. Otros como Bermejo-Acosta (2003) se centran en las nuevas prácticas comunicativas que han dado lugar a nuevas dinámicas de medición. En este sentido, es de resaltar la reflexión de Aguado-Guadalupe (2017: 145) cuando matiza que "en el entorno digital el debate e investigación en métricas de audiencia se centra en torno al tipo de conocimiento para poder valorar la reacción del público frente a los contenidos y poder cuantificarlos y rentabilizarlo". Especial atención ha merecido la incidencia de los usuarios en las métricas. Ello ha llevado a Papí y Escandell (2014) a reflexionar sobre la precisión que se requiere a la hora de catalogar los públicos, dejando de priorizar los rasgos sociodemográficos para centrarse en el comportamiento, de manera que "se impacta solo en los usuarios que hayan realizado una actividad online concreta" (Papí y Escandell, 2014: 61).

Los cambios acontecidos en el ámbito digital han llevado a cuestionar incluso el concepto de audiencia, así como el conocimiento requerido sobre la misma para desarrollar estrategias de contenido. En este sentido Huertas (2006: 201), ha planteado que "la pregunta correcta no sería ¿es posible conocer la audiencia?, sino ¿qué tipo de conocimiento es posible y necesario?". Dicho concepto de audiencia ha merecido reflexión por parte Garay-Cruz (2006: 9) quien resalta "la imposibilidad de que la mal llamada masa pueda reaccionar de manera uniforme cuando realmente está compuesta por diversos grupos y por sujetos con diferencias psicológicas individuales".

Además, se han contemplado nuevos aspectos a considerar en la medición de audiencias, como las redes sociales, que han merecido la atención de Echegaray y Peñafiel (2013). También se ha observado que "el protagonista de este escenario digital e interactivo es alguien que opina, se compromete y domina -en la gran mayoría de las ocasiones- el lenguaje del marketing y la publicidad" (Del Pino y Galán, 2010: 56). Todo ello en un entorno en el que "los mensajes están siendo sustituidos por contextos de comunicación en torno a las marcas, con los que atraer e interesar a un nuevo tipo de consumidor más crítico y formado en medios y contenidos, que participa de forma interactiva y proactiva" (Martín-Parreño, 2006: 6). A este respecto, Aparici y Osuna (2013: 139) indican que "mientras la interactividad es una propiedad que proporciona la tecnología, la participación es una propiedad que proporciona la cultura".

En dicho entorno se ha abierto paso la medición de la denominada audiencia social, que ha sido abordada por autores como Quintas-Froufe y González-Neira (2014) que han reflexionado sobre los elementos que contribuyen al éxito o fracaso de programas con un mismo formato en relación a la audiencia social, entendida como "una fragmentación de la audiencia real en función de su interactividad en las redes (Quintas-Froufe y González-Neira, 2014: 83). Este aspecto ha merecido también la reflexión de Congosto; Deltell; Claes; Florencia y Osteso (2013: 78), quienes han llegado a la conclusión de que "si bien Twitter no puede sustituir al sistema audimétrico sí lo complementa".

En el análisis de esa audiencia social han adquirido especial protagonismo los influencers (Hornos, 2015), cuya identificación es un reto, así como la medición de su impacto. Se ha considerado igualmente la importancia de las herramientas que evalúan dicha influencia (Serrano-Puche, 2012). Ello ha merecido reflexiones como la publicada por Adit Aunit (2009) y estudios como el de Max Planck Institute for Sofware Systems en el marco del Twitter Projects (Chat; Haddadi; 
Benevenuto y Gummadi, Krishnap, 2010), llegando a desmentir mitos sobre el papel que ejercen de forma aislada en la influencia variables como la popularidad o el número de seguidores, poniendo de relieve la necesidad de combinar varios factores para medirla. En esta línea es de destacar la aportación de Launchmetrics (2017) con el Informe sobre el estatus del marketing de influencers.

Estos nuevos aspectos considerados en las métricas brindan la posibilidad de aplicar técnica de marketing no intrusivas para captar seguidores, clientes y prescriptores aportando valor (Pintado-Blanco y Sánchez-Herrera, 2014). Todo ello en un entorno donde se ha dejado de lado la agresividad y el intrusismo, sustituidos por el permiso (Godin, 1999). Estamos en realidad, como señala Meerman Scott (2007) ante un resultado de la propia evolución del marketing en el ecosistema que las herramientas 2.0 han ido creando en Internet, donde el marketing emocional está adquiriendo un papel predominante (Cadavid-Gómez, 2004).

\section{Metodología}

Para realizar el presente trabajo se ha aplicado una metodología de carácter cualitativo y descriptivo. Se ha partido del análisis de herramientas de medición de audiencias como Social Mention, SWB Social, Agora Pulse, Google Analytics, Twiter Analytics, Brandwatch Analytics, Brandwatch Audiences. Al tiempo que se ha realizado un seguimento de los servicios que ofrece ComsCore. Dichas herramientas han sido seleccionadas en tanto que los datos que aportan permiten acercarse al conocimiento de los comportamientos del público con respecto a los contenidos, así como hacer un seguimiento del rendimiento medio de cada publicación, las frecuencias de visita, la audiencia compartida y el engagement.

Para la realización del trabajo se han analizado también las aportaciones y avances que se están realizando por parte de algunas empresas en el ámbito de las métricas de sentimientos. Para ello se ha recurrido al seguimiento de las prácticas desarrolladas por parte de entidades como Sociograph y Neuromarketing Labs. En ambos casos se ha observado las técnicas que se utilizan para hacer el seguimiento de los sentimientos que provocan los contenidos en el público. También se ha analizado el diseño de los estudios que realizan, así como las variables que miden y el tipo de pruebas que se llevan a término.

Con el análisis de las diferentes herramientas y metodologías se ha buscado identificar las principales métricas de audiencia en lo que se refiere a comportamientos y sentimientos de los públicos y que inciden en el desarrollo de estrategias de inbound marketing y muy especialmente de estrategias de marketing de contenido.

\section{Resultados}

\subsection{Métricas de comportamiento e influencia al servicio del Inbound Marketing}

Para el conocimiento de los comportamientos del público con respecto a los contenidos en el entorno digital se cuenta con diferentes servicios y herramientas de medición, que permiten, según los casos, la monitorización de contenidos y marcas en tiempo real, la medición de rendimiento, el seguimiento de sentimientos, las frecuencias de contacto, la distribución del tráfico, el engagement y la influencia. Tal es el caso de: 
a. Social Mention, que permite monitorizar en tiempo real quién menciona un contenido o marca, dónde y cuándo, teniendo en cuenta cuatro parámetros: 1) fuerza: posibilidad de que se hable sobre un contenido o marca; 2) sentimiento: mide la influencia de los comentarios positivos frente a los negativos; 3) pasión: probabilidad de que un usuario único hable de un contenido o marca; y 4) alcance: nivel de influencia del contenido o marca. Para ello aporta datos como: a) sentimientos positivos, negativos y neutros; b) qué palabras clave tienen más impacto en la búsqueda y menciones; c) los usuarios que más menciones realizan; d) los principales hashtags con los que se asocian el contenido o marca en las menciones; $\mathrm{y}$ e) las principales fuentes de menciones.

b. SWB Social, de Infotec, que permite hacer un seguimiento de los sentimientos en tiempo real en prácticamente todas las redes sociales. Es capaz de identificar cuándo un comentario es positivo, negativo o neutro. Estos comentarios se integran en un diccionario de términos y expresiones comunes en redes, que incluye emoticonos, de manera que la aplicación identifica sentimientos, influencia, geolocalización e idioma.

c. Agora Pulse, que es una herramienta que permite ver el rendimiento medio de cada publicación, apreciando el alcance medio y el número de interacciones. Además, Agora Pulse Barometer permite conocer si una página rinde de manera adecuada utilizando indicadores como fans alcanzados, engagement, feedback negativo, alcance viral, alcance orgánico, y click through rate.

d. Google Analytics, que ofrece analítica de contenidos viendo con qué frecuencia se visita cada página, tiempo de visita y frecuencia de conversiones. Mide conversaciones observando las ventas, las descargas, las reproducciones de video y el retorno.

e. ComScore, que además de otros indicadores, proporciona información sobre el porcentaje de audiencia que se comparte con otro sitio web del mismo sector de actividad. También indica sitios web que han visitado los usuarios antes y después de navegar por una web y posibilita apreciar cómo se distribuye el tráfico entre las distintas secciones de un sitio web.

f. Twitter Analytics, que permite medir en engagement a través de la tasa de interacción, los clics en el enlace, los retweets, favoritos y respuestas.

g. Brandwatch Analytics y Brandwatch Audiences, que aportan métricas de influencia al considerar indicadores como: me gusta, compartir, comentarios, personas alcanzadas y engagement. También, permiten realizar una labor de localización, identificando el lugar donde se está hablando sobre un término buscado y un análisis de sentimientos, separando las palabras negativas de los elogios.

h. Demographic Insights, que permite analizar el tipo de cuenta, género, profesión e intereses.

\subsection{Métricas de sentimientos basadas en neuromarketing}

Según el informe de GlobalwebIndex (2017) en Estados Unidos el perfil que más bloqueadores tiene está entre 25 y 34 años, aunque sólo el $15 \%$ lo tiene en el móvil frente al $22 \%$ que lo tiene en el ordenador y el portátil. En edades más tempranas los bloqueadores de publicidad están más presentes en los teléfonos móviles. Por ello, las marcas buscan estrategias para conectar con las personas. Al mismo tiempo, se están introduciendo intermediarios tecnológicos más directos y emocionales como los asistentes inteligentes (Siri, Cortana, Alexa...) que buscan una relación más directa con el individuo. 
Johnston y McQuivey (2017) indican la importancia de crear un contexto y una conversación que generen una historia emocional con el cliente para conseguir fidelización. Según el estudio de fidelización The Loyalty Report 2017 elaborado por Bond Brand en asociación con Visa, cuatro consumidores de cada cinco operan con las marcas si se les atrae, mientras que dos tercios de ellos modifican la inversión en otras marcas para maximizar ventajas de lealtad, y tres cuartos de los encuestados afirmaron que recomendarían marcas con buenos programas de lealtad. Esto se debe, según Manovich (2017:60), a que estamos ante la primera ocasión de describir, modelar y simular el universo cultural completo replanteándonos conceptos básicos en la nueva relación entre marcas y consumidores.

Manovich (2017) plantea que estamos ante la posibilidad de trabajar con patrones predictivos a partir del dato captado y almacenado. Por ello, las métricas aportan valoraciones finales que conducen al resultado por tendencias y no por actuaciones según las mediciones tradicionales. Este sistema de medición basado en probabilidades recoge aspectos como la atención y la emoción que, tradicionalmente, han escapado de la valoración más directa de los sistemas conductivos de medición. En este sentido, la emoción es un factor que altera la forma en la que se reescriben las intenciones estratégicas porque toda predicción necesita de un planteamiento previo que, a su vez, demanda de una simulación.

El sistema de integración del usuario en una red de datos procedentes no sólo de su interacción dentro de las redes sociales, sino de cualquier instrumento con capacidad de conectarse a Internet, convierte la analítica de datos en una herramienta escénica donde los aspectos pragmáticos (geolocalización, horas, tiempos de consumo,) confluyen con el aspecto emocional de la interacción. Los datos dejan de ser lineales y escalables y se trasladan a un mapa de acción, interacción y representación (Gil de Zúñiga; García-Perdomo; McGregor, 2015).

No obstante, Felt (2016) indica que los investigadores deben comprender las limitaciones del análisis de datos, al tiempo que existen limitaciones en la captura de tal información. Mayer (2016) reitera este punto explicando que durante los últimos treinta años, la recepción de medios de comunicación ha buscado trabajadores, amas de casa y adolescentes para comprender las formas en que los grupos interpretan los textos mediáticos y también para interpretar la respuesta a las técnicas de medición de audiencias.

Pero existen autores que indican que hay que cuantificar la respuesta al texto (Wood, 2009), o las reflexiones que el público articula en relación al contenido consumido (Sender, 2012) para alcanzar otra dimensión que cuantifique la experiencia más que el hábito, en lo que se conoce como audiencia social. Ello deriva en aspectos cualitativos que influyen en la percepción del grupo en torno al objeto de la medición.

Así, la atención y la emoción adquieren un papel esencial en los retornos de inversión en los contenidos que se articulan a través de Internet, por lo que hay que medirlo y convertirlo en dato para analizarlo y transformarlo posteriormente en información. De ahí que el neuromarketing o marketing emocional resulte esencial para optimizar las nuevas métricas en los contenidos audiovisuales digitales.

Importante en este sentido son las aportaciones de entidades como Sociograph y Neuromarketing Labs. En el caso de Sociograph, a través del método de corriente constante, evalúa la atención y emoción de grupos de personas ante el impacto de estímulos, con lo que se consigue medir y calibrar de manera cuantitativa la actividad grupal. La tecnología consiste en registrar simultáneamente la actividad electrodérmica (EDA) de un número de sujetos - que puede llegar hasta ciento 
veintiocho-, y cuyas señales son transmitidas, con un dispositivo colocado en la mano, de forma inalámbrica y analizadas de forma sincronizada una vez recibidos, en una unidad central.

Tras esto, los datos son analizados y procesados matemáticamente dentro de una metodología de análisis de series temporales mediante la aplicación de algoritmos específicos. Las variables que se miden son la EDL (electrodermal level), actividad tónica asociada a la atención, y la EDR (electrodermal response), con el que se computa la actividad fásica relacionada con la emoción, variables que permiten realizar un cálculo de la efectividad de los contenidos en tres instantes puntuales: antes de ser lanzados, en fase piloto y, posteriormente, una vez lanzados. No obstante, existe una prueba de test previa en modo de focus group cuya actividad también es registrada en dos direcciones: análisis de contenido basado en la técnica del propio método y registro de la actividad electrodérmica mediante la aplicación de Sociograph.

En este sentido, cualquier estudio es diseñado en función de tres fases de trabajo que permiten obtener resultados eficaces en relación a la métrica de sentimientos. Así, una primera fase denominada NeuroDesign, en la que cuantifican la efectividad de cada elemento con el fin de determinar la eficiencia de cada uno de ellos; la segunda fase consiste en el NeuroPretest, en el que se comprueba la efectividad de la comunicación una vez que se ha diseñado pero que no ha sido lanzada para poder incluir modificaciones que mejoren los resultados. La última fase de trabajo se conoce como NeuroMetrics, que permite cuantificar el impacto y la reacción de la audiencia ante cualquier comunicación.

En definitiva, para esta técnica conocida como Sociograph, la respuesta más óptima o el índice de rendimiento ideal ante un estímulo es aquella que consigue despertar la atención y crear un vínculo emocional fuerte.

Neuromarketing Labs, otro ejemplo de empresa dedicada al análisis de métricas de sentimientos a partir de investigación neurocientífica, basa su acción en algoritmos creados por ellos que permiten hacer predicciones de alta precisión en torno a consumidores. Para ello emplean técnicas como electroencefalograma (EGG), que posibilita medir la actividad cerebral de manera precisa y en tiempos de milisegundo, y la resonancia magnética (fMRI) que permiten localizar la actividad cerebral con total precisión. Ambas técnicas conjugadas permiten comprender el comportamiento del usuario de manera fiel, aunque en algunas ocasiones complementan los estudios con técnicas como el eye-tracking o mediciones de tipo biométrico y psicológico.

Con la EGG se mide el impacto inmediato que un estímulo provoca en el cerebro en función de la actividad desarrollada que, por otra parte, puede ser filmada y recreada, además de ser una técnica no invasiva. El fMRI (Functional Magnetic Resonance Imaging) es un método aplicado a medir emociones cuya efectividad es superior a los cuestionarios tradicionales, según indican autores como Berns y Moore (2011).

Con la técnica del eye-tracking se estimula la córnea para seguir la dirección de la mirada y, de esta manera, se puede medir el interés concreto y la atención de una persona en una determinada imagen. Además, se estipula una codificación por colores de las zonas de mayor y menor interés dentro de la imagen. Las técnicas biométricas, que son utilizadas como una herramienta habitual de medición en apps centradas en temas de salud, sirven para medir aspectos como la actividad electrodermal (EDA), la tasa de respiración, el ritmo cardíaco, la actividad muscular en electromiograma (EMG), etc., que son variables influidas directamente por la emoción y cuyos resultados sirven para calibrar en tiempo real los efectos de un contenido. 
Además, todas estas variables pueden ser relacionadas con métodos de análisis psicofísicos que complementan el estudio de factores como el tiempo de reacción ante un estímulo, la atención o factores de decisión, etc. El objetivo es ayudar a comprender el comportamiento de un consumidor ante estímulos emocionales proporcionados en las diferentes campañas. Las tres variables que se miden son la atención, la emoción y la memoria.

Para la medida de la atención se utilizan las técnicas citadas anteriormente para llegar a obtener valores sobre la atención captada a un consumidor frente a un producto identificando aspectos específicos del mismo. Para la emoción, esta empresa la analiza bajo una doble perspectiva: aquella que la relaciona con la evocación a aspectos positivos y/o negativos (EGG) y aquella que relaciona la emoción con aspectos como la alegría, deseo, confianza, desconfianza, familiaridad, miedo, temor o disgusto (fMRI). En lo referido a la memoria, la dividen en dos tipos según su influencia en el comportamiento de compra de los consumidores: la memoria explícita y la memoria implícita. La primera implica hechos recuperables conscientemente (eslogan, direcciones web,...), mientras que la memoria implícita implica la retención inconsciente, es decir, aquella que relaciona el producto con el entorno del consumidor.

El proceso de trabajo que sigue Neuromarketing Labs pasa por una primera fase en la que definen el grupo objetivo para el producto o servicio específico, reclutando el grupo de sujetos específicos que definen los principios sobre los que se va a sustentar el estudio. Los algoritmos desarrollados por la empresa facilitan un análisis de datos exhaustivo que permiten recomendar al cliente actuaciones específicas y un plan de acción. El elemento común integrador es el empleo de técnicas específicas de estudio del cerebro y del comportamiento asociados al consumo de imágenes. Esta nueva forma de entender el estudio de las emociones en marketing está derivando en una metodología cuantitativa -basada en el análisis de datosque parte de un factor cualitativo como es el sentimiento o la emoción, así como de variables, también cualitativas, que con metodologías previas eran difícil de cuantificar y se ajustaban a la intuición de los creativos y estrategas de campañas.

Por tanto, el mayor avance consiste en la cuantificación de insights cualitativos implícitos en cualquier contenido. Este principio de determinismo numérico implica una mayor capacidad de análisis en tiempos cada vez más cortos y, por otro lado, un aislamiento respecto a variables externas (como el recuerdo, principal factor de validez respecto al modelo de encuesta que se ha usado tradicionalmente en las metodologías de audiencia) condicionantes del resultado final en la medición de la eficacia y que, por otro lado, no atienden variables concretas como la emoción y atención que implica el propio visionado en tiempo real.

Por otra parte, el uso de estos indicadores habilita nuevas posibilidades relacionados con la automatización y agilidad de los procesos, obteniendo resultados más fiables y optimizados para satisfacer intereses individuales. Todos los datos deben ser escalados en la medida que requiera la atención del usuario, debiendo ser focalizados en resultados directos y acertados. La analítica de datos ofrece alternativas en tiempo real que proporcionan la posibilidad de una interacción directa y automática mediante estímulos que provoquen al interés y la emoción del individuo.

Ha de considerarse que la métrica de sentimientos no se articula sólo en relación a datos concretos de consumo, sino que se introducen otros indicadores como el texto, imágenes y vídeos del propio usuario, datos estructurados en función de contactos, apps descargadas, etc., que son indicadores que contextualizan las preferencias de consumo y el destino de 
las mismas hacia el entorno social de cada individuo. Todos estos datos capturados y representados en métricas ayudan a crear modelos que definen algoritmos que señalan el comportamiento de cada individuo.

De ahí que es necesario establecer técnicas metodológicas con capacidad de discriminación particular de cada individuo en función de su comportamiento en el entorno social del universo digital. Nadie podía suponer hace unos pocos años que estuviéramos tan cerca de medir el comportamiento y la emoción mediante técnicas de medición únicas. Al mismo tiempo, es relevante la consideración de que se aplican en el análisis y la toma de decisiones en tiempo real y de manera personalizada para cada individuo activo en cada instante de la campaña. Estas decisiones, matemáticamente creadas, y que afectan a la interrelación del consumidor con el producto y viceversa, obliga a establecer lazos emocionales de la marca con el cliente, las cuales deben afianzarse en cada acción.

Es curiosa la relación que se establece, a través del marketing de los sentimientos, entre el análisis de datos numéricos y la emoción y/o la atención. Estas últimas variables siempre han resultado fundamentales para determinar el éxito o el fracaso de una campaña de marketing o de publicidad, así como de cualquier tipo de contenido audiovisual. Las métricas de sentimiento basadas en neuromarketing no miden el impacto en grandes masas de población, sino la influencia emocional que afecta al individuo. Para ello se trabaja mediante grupos de interacción en los escenarios pensados para cada contenido (salas de cine, teatros, salas de trabajo para investigación mediante focus group, etc.), creando inmensas cantidades de datos que son tratados de manera personalizada y en relación al grupo.

De esta manera, se obtienen garantías de éxito respecto al valor real del estudio y sirve para modificar todos aquellos aspectos imprecisos o no relevantes para entender el éxito y la eficacia en la construcción del contenido.

\subsection{Incidencia de las métricas en la oferta de contenidos}

Steemeres (2016) especifica cómo la distribución digital facilita que los usuarios tengan libertad de engancharse al contenido en cualquier tiempo y lugar, sobre dispositivos electrónicos que rompen el concepto de espacio superando la tradicional relación entre contenido y medio. En los contenidos audiovisuales se produce una influencia mayor por los estados conversacionales, ya que la lógica de compartir y recomendar es una cualidad imprescindible e inherente de las redes sociales (Groshek, 2016). De ahí que una de las características que define el nuevo modelo de consumo de contenidos esté basada en la participación activa de los usuarios a través del visionado simultáneo en segundas pantallas. Con estos contenidos personalizados y dirigidos a públicos objetivos, se busca compartir experiencia social a través de la interacción en las redes.

A ello se une una nueva manera de entender el consumo que, como indica Álvarez-Monzoncillo (2011), consiste en un conjunto de agregadores, como Netflix o Amazon Prime, transformados en tiendas virtuales con un entorno virtual de intermediación y compra de contenidos dentro de la nube y consolidado como la manera ideal de producir interrelaciones entre productores, distribuidores y usuarios en cualquier tiempo y en cualquier espacio.

En este sentido es conveniente entender que la base metodológica de algoritmos tan famosos y de tanto éxito como el que soporta una empresa como Netflix se basa en convertir valoraciones cualitativas de los usuarios en análisis cuantitativos. Así, según se explica en Hallinan y Striphas (2016: 120), el procedimiento que emplea la exitosa empresa de distribuidora 
de contenidos a escala global se basa, como principio de adquisición de datos sobre cada producto, en la valoración de los clientes sobre cada contenido que disfruta a través de una valoración, en todo momento, puramente emocional, basada en una escala que va de una a cinco estrellas en la que cada estrella tiene una relevancia de valoración en el contexto general. Así, cinco estrellas es "Me encanta", cuatro se refiere a "Realmente me gusta", tres a "Me gusta", dos estrellas a "No me gusta" y una estrella a "Lo odio".

A continuación y en función del valor cuantitativo asignado a la cantidad de estrellas de valoración, establece un análisis cuantitativo que discrimina, casi de manera personalizado, las preferencias de consumo de cada cliente.

Por ello, Lotz (2007) argumenta que no tiene sentido hablar de audiencia, siendo preferible hablar de nicho, en un contexto en el que se extrapolan los datos de las conversaciones sociales basados en intereses y gustos por temáticas de contenidos para crear productos de éxito que aportan liderazgo en las campañas de marketing. Si bien, los ejemplos paradigmáticos los encontramos en las series propias producidas por Netflix, que son reflejo de lo que consumen sus usuarios sistematizados por franjas de edad, nivel socioeconómico, etc., más las interacciones en redes sociales que provoca el consumo y los intereses específicos generados a partir de las temáticas.

Otro caso concreto lo encontramos en la campaña del Banco Santander "Cuenta. Más allá del dinero". El objetivo era captar a la generación millenials caracterizada por primar las experiencias vitales sociales. Ambientada en un mundo futurista donde las vivencias se convierten en códigos numéricos que pueden venderse o comprarse, es un ejemplo paradigmático del uso de la emoción como un dato cuantificado que incrementa la capacidad de afección del usuario al universo digital.

Es interesante comprobar cómo la experiencia del visionado está asociada a la visión tecnológica del nicho sobre el que quiere incidir la campaña. Además, el nombre del cortometraje coincide con el de la cuenta bancaria objeto de la promoción, que reitera el perfil grupal al que se dirige en elementos como el escenario urbano limpio y elegante, el nivel económico ideal, la filosofía de vida, etc. Aspectos estudiados a partir de las métricas generadas en las interacciones de redes sociales y a las que el Banco Santander accede gracias a su apuesta por la financiación universitaria con Universia.

En esta línea, el grupo Mediaset ha recurrido a Sociograph para realizar métricas de sentimientos en caso de series de producción propia como El Príncipe y $B \& b$. Se ha medido la eficacia para diferentes campañas de publicidad, películas o también estudios dentro del Festival de cine de la Seminici de Valladolid. Es una apuesta continua por el mensaje emocional que transmite la imagen en movimiento y que tan relevante se ha convertido en la creación de la imagen de marca de cualquier empresa, producto o contenido.

Por su parte, la empresa Neuromarketing Lab ha prestado sus servicios de métricas en series de ficción como Breaking Bad y otras de la cadena Fox. Pero también son clientes empresas del calado de Bayer, Volkswagen, Audi, Roche, Hussel y Pepsico, que demandan eficacia y éxito en sus campañas de comunicación dadas las abultadas cifras económicas que se manejan.

La eficacia ha pasado a depender, por tanto, de un análisis predictivo riguroso a partir de factores y variables condicionados por las características específicas del consumo digital. Esto es, la interacción que debe ser medida se realiza a partir de aspectos y variables físicas (redes sociales, geolocalización, tiempos de consumo, etc.) así como de aspectos emocionales 
que parecen estar perfectamente integradas en las nuevas métricas. Nos encontramos ante lo que se conoce, según Serrano-Puche (2017: 79) "como la "economía de la atención" donde los productores de contenido (y, en general, todo aquel que quiere comunicar algo) compiten por captar el interés de las personas, buscando ocupar su tiempo disponible”.Y este nuevo escenario debe ser cuantificado de manera ajustada a las variables que lo definen y configuran.

En dicho contexto, las audiencias han pasado de ser una masa a estar formada por individuos con características específicas. Dichas características pueden ser cuantificadas tanto en atención a variables concretas (geolocalización, contactos, uso de redes, etc.) como a variables dispersas (basadas en aspectos relacionados con la manera de ser de cada individuo, tanto en sus relaciones personales como en el entorno que le rodea). En este nuevo universo propio de la era digital, las tendencias son construidas a partir de las emociones y la capacidad de generar atención, aspectos que las marcas empiezan a dominar y considerar como variables diferenciadoras y potenciadoras de éxito frente al resto.

\subsection{Medición de conversión, alcance social y monitorización del comportamiento del usuario}

Partiendo de que el inbound marketing se compone de tres elementos: 1) contenidos de interés; 2) estrategias de marketing para atraer tráfico a las páginas y 3) capacidad de educar al usuario para que sea receptivo a la oferta, se puede decir que en el desarrollo de estrategias de inbound marketing no sólo es importante generar tráfico a través de contenidos que aporten valor, sino también medir la conversión, es decir el paso de visitas a lead y de lead a clientes. Se precisa igualmente conocer el alcance social, ya que proporciona un tráfico adicional, y da indicación de si se está compartiendo el contenido. Para ello se requiere poder valorar la calidad de cada usuario monitorizando su comportamiento con respecto al contenido.

Para dicha monitorización y seguimiento se ha observado que en los diferentes servicios de métrica y herramientas utilizadas para ello adquieren una relevancia especial indicadores como:

a) Interacciones (menciones, comentarios, veces que el contenido es compartido).

b) Consumo (vistas de página, sesiones, tiempo por página, páginas por sesión, rebotes, visualizaciones de vídeo, retorno de lectura, frecuencia de visita en un periodo temporal, correos abiertos, clics en emails, CTR).

c) Conversión (petición de demo, descarga de contenidos, suscripciones, formularios).

d) Canales (según se llegue al contenido a través de buscadores, directamente a través de la URL, haciendo clic en link compartidos en redes, desde otras páginas que no son buscadores, a través de anuncios o por correos que han recibo).

e) Ranking de palabras clave.

f) Enlaces inbound (que miden la eficacia de conseguir que otros hablen de ti).

g) Engagement (orientadas a la tasa de viralidad, orientadas a medir la tasa de likes y orientadas a la conversión).

h) Influencia (número de conversaciones sobre un tema, comentarios en el contenido creados por el influencer, total de influencers que publican sobre un contenido o marca, tráfico referido proveniente de los contenidos del influencer, y conversiones atribuidas a la publicación de influencer.

A todo ello se unen las denominadas métricas de sentimientos, que ofrecen la posibilidad de medir la emoción de manera cuantitativa y traducirla en datos relevantes y objetivables, hecho fundamental para afrontar con éxito la eficacia publici- 
taria. Desde siempre, la publicidad y la emoción han estado íntimamente ligadas en función de diferentes variables como la cultura, sexo, etc., que han definido el perfil del público demandado. Deza (2012: 10) explica que:

"las emociones no nacen, sino que son parte de un sistema automatizado que nos permite reaccionar ante el mundo, de
una forma inmediata y sin necesidad de pensar, con el cual ya venimos dotados desde el nacimiento. Las emociones forman
parte de esta compleja maquinaria en la que intervienen las recompensas y los castigos, el estímulo y la emoción... y todo
aquello que hace que deseemos comer, beber, practicar sexo... Las emociones son parte del proceso de la regulación de un
cuerpo vivo, y se presentan con diferentes formas y sabores".

Hay unas emociones primarias y sencillas como son el miedo, la rabia, la felicidad o la desdicha... Hay emociones sociales, más complejas, como la compasión, el desprecio, la admiración, el orgullo...”. La emoción es, en consecuencia, y tal como se recoge en Gutiérrez (2005), una respuesta a una sensación que puede ser consciente o inconsciente que, en todo caso, puede ser estimulada y medida al poseer naturaleza física. Estas dos variables están determinando una manera diferente de articular métricas en torno a efectos físicos medibles que influyen en los resultados de retorno de las campañas y que condicionan, inexorablemente, las formas de construcción del discurso basado en la acción y la emoción. Aquí se encuentra el punto de avance de la era digital sobre épocas anteriores y es aquí donde la interacción se define como parte esencial del mensaje. El usuario actúa y se emociona y el creador lo mide y toma decisiones al respecto.

La consideración de estas nuevas variables en las métricas viene promovida por la forma en que las generaciones recientes - millenials principalmente- están relacionándose con los medios, modificando radicalmente la relación con los mismos, convirtiendo a la publicidad en acciones con capacidad de crear fidelización a través de aspectos vitales como la emoción. Dando lugar a un escenario en el que es necesario converger hacia dinámicas de trabajo en las que dicha variable se convierta en un indicador más de la campaña, llegando incluso a valorarse como un condicionante de construcción del mensaje.

Cuadro 1: Herramientas, métricas e indicadores para Inbound Marketing

\begin{tabular}{|c|c|c|}
\hline Herramientas & Tipo de métrica & Indicadores en relación al Inbound Marketing \\
\hline Social Mention & $\begin{array}{l}\text { Comportamiento } \\
\text { Sentimientos }\end{array}$ & \begin{tabular}{|ll}
- & Fans \\
- & Consumo \\
- & Interacción \\
- & Alcance \\
- & Ránkings palabras clave \\
- & Engagement
\end{tabular} \\
\hline SWB Social & $\begin{array}{l}\text { Comportamiento } \\
\text { Sentimientos }\end{array}$ & $\begin{array}{ll}- & \text { Sentimientos } \\
- & \text { Influencia } \\
- & \text { Geolocalización } \\
- & \text { Idioma } \\
- & \text { Consumo } \\
- & \text { Interacción } \\
- & \text { Engagement }\end{array}$ \\
\hline
\end{tabular}


Nuevas métricas de audiencia al servicio del Inbound Marketing

\begin{tabular}{|c|c|c|}
\hline Com Score & Comportamiento & \begin{tabular}{|ll}
- & Consumo \\
- & Secuencia de consumo \\
- & Distribución de tráfico por secciones \\
- & Influencia \\
\end{tabular} \\
\hline Google Analitics & Comportamiento & $\begin{array}{ll}- & \text { Consumo } \\
- & \text { Interacción } \\
- & \text { Engagement } \\
- & \text { Influencia } \\
- & \text { Conversión } \\
\end{array}$ \\
\hline Twiter Analitics & Comportamiento & \begin{tabular}{|ll}
- & Consumo \\
- & Interacción \\
- & Engagement \\
- & Influencia \\
\end{tabular} \\
\hline $\begin{array}{l}\text { Brandwatch Analytics y } \\
\text { Brandwatch Audience }\end{array}$ & $\begin{array}{l}\text { Comportamiento } \\
\text { Sentimientos }\end{array}$ & $\begin{array}{ll}- & \text { Consumo } \\
- & \text { Interacción } \\
- & \text { Engagement } \\
- & \text { Influencia } \\
- & \text { Localización } \\
- & \text { Identificación de palabras } \\
\end{array}$ \\
\hline Demographic Insight & Comportamiento & \begin{tabular}{|ll}
- & Consumo \\
- & Cualificación audiencia \\
- & Cuenta \\
- & Datos demográficos \\
- & Conversión \\
\end{tabular} \\
\hline Agora Pulse & Comportamiento & \begin{tabular}{|ll}
- & Consumo \\
- & Interacción \\
- & Influencia \\
- & Engagement \\
- & Conversión \\
- & Enlaces Inbound \\
\end{tabular} \\
\hline Socciograph & $\begin{array}{l}\text { Sentimientos: } \\
\text { Emoción (EDL) } \\
\text { Atención (EDR) }\end{array}$ & $\begin{array}{ll}- & \text { Actividad electrodérmica (EDA) } \\
- & \text { Actividad física } \\
\text { - } & \text { Análisis de contenido } \\
\text { - } & \text { Focus Group, NeuroDesign, NeuroPretest, NeuroMetrics } \\
- & \text { Îndice de rendimiento y atención } \\
- & \text { Creación de vínculo emocional fuerte } \\
\end{array}$ \\
\hline Neuromarketing Lab & $\begin{array}{l}\text { Comportamiento } \\
\text { Sentimientos }\end{array}$ & $\begin{array}{ll}- & \text { Electroencefalograma (EGG), Resonancia magnética (fMRI) } \\
- & \text { Eye tracking: mediciones biométrico y psicológico } \\
- & \text { Tiempo de reacción ante el estímulo } \\
- & \text { Atención } \\
- & \text { Factores de decisión } \\
- & \text { Cuantificación de } \text { inshights cualitativos } \\
- & \text { Influencia emocional }\end{array}$ \\
\hline
\end{tabular}

Fuente: Elaboración propia

\section{Conclusiones}

Frente a la tradicional cuantificación de volúmenes de audiencia, tiempos de consulta y aspectos geográficos y demográficos, las nuevas métricas de audiencia surgidas en el entorno digital se centran esencialmente en el análisis comportamental y emocional de los usuarios, proporcionando información sobre la interactuación de los mismos con los con- 
tenidos y aportando datos que son tenidos en cuenta precisamente en el proceso de elaboración de dichos contenidos, considerando las necesidades detectadas en el feed back generado.

En consonancia con la hipótesis de partida, tras observar los resultados obtenidos, se puede afirmar que en el entorno digital las métricas se han convertido en herramientas indispensables, que permiten identificar la capacidad de atracción, retención, fidelización y satisfacción de los usuarios frente a los contenidos. La analítica de datos ha pasado a ser una herramienta en la que los aspectos pragmáticos se unen con aspectos emocionales, permitiendo cuantificar la experiencia y la emoción más allá del hábito. De manera que la atención y la emoción adquieren un papel esencial en el retorno de inversión en los contenidos. Por su parte, las métricas comportamentales permiten además realizar predicciones, posibilitando planificar los contenidos para adaptarlos a los requerimientos de los usuarios.

Dichas métricas se han convertido en elementos esenciales para el desarrollo de prácticas comunicativas de inbound marketing, en tanto que posibilitan contar con información esencial tanto para el diseño de contenidos como para su distribución. Gracias a ellas se pueden detectar no sólo necesidades en el mercado, sino también conocer el trayecto o trazabilidad del usuario.

El conocimiento que brindan las métricas permite considerar múltiples variables que deben sincronizarse e interactuar entre sí en el momento adecuado, brindando la posibilidad de generar contenidos no intrusivos, que aporten en muchos casos soluciones y respuestas, logrando los tres objetivos de toda acción de inbound marketing: atraer, retener y fidelizar al público.

Así pues, en este nuevo paradigma cuantitativo será esencial establecer criterios únicos y personalizados adaptados al contenido y al espectador. Para ello, tanto las métricas de comportamiento como de sentimiento se convertirán en un referente crucial, en tanto que permiten conocer la manera en la que cada individuo se enfrenta y reacciona a un mensaje.

Por tanto, las nuevas métricas deberán centrarse en encontrar métodos de análisis que confluyan en un nuevo escenario donde los aspectos fundamentales sean insights basados en datos que permitan establecer protocolos de medición personalizados. Al tiempo que estén configurados para posibilitar la toma de decisiones en la elaboración y distribución de contenidos adaptados a la personalidad, necesidades, deseos y circunstancias de los individuos y donde además se consideren aspectos como la atención, la emoción, el recuerdo y la interacción.

\section{Referencias bibliográficas}

Aguado-Guadalupe, G. (2017): "Repercusión de las métricas de audiencia online en la comercialización publicitaria de los productos informativos”, Razón y Palabra, vol. 21, n. 2-97, pp. 142-158. Disponible en: https://goo.gl/8r7uS8 [Consultado el 10/12/2017].

Aguado-Guadalupe, G. (2015): “Inbound Marketing en Linkedin para la gestión de marca”, Icono 14, vol. 13, n. 1, pp. 105124. Disponible en: https://goo.gl/tFSmF3. [Consultado el 11/09/2017]. DOI: 10.7195/ri14.v13i1.741.

Álvarez-Monzoncillo, J. M. (2011): Watching the Internet: The Future of TV? Formalpress, Media XXI.

Banco Santander (2017): Sala de Comunicación. Disponible en: https://goo.gl/wNukcC [Consultado el 11/09/2017].

enero-junio de 2018

doxa.comunicación | n²6, pp. 81-98 | 95 
Berns, G.S. y Moore, S. E. (2011): “A neural predictor of cultural popularity”, Journal of Consumer Psychology, vol. 22, n.1, pp. 154-160.

Aparici, R. y Osuna Acedo, S. (2013): La Cultura de la Participación. Revista Mediterránea de Comunicación, vol. 4, n. 2, 137-148. Disponible en: https://goo.gl/xnK9zq. [Consultado el 11/09/2017]. DOI: 10.14198/MEDCOM2013.4.2.07.

Barroso Castro, C. y Martín Armario, E. (1999): Marketing Relacional. Madrid: Esic.

Bermejo-Acosta, F. (2003): La medición de audiencias en Internet: orígenes, métodos y dinámicas de la industria (Tesis Doctoral). Facultat de Ciències de la Comunicació, Departament de Comunicació Audiovisual i Publicitat. Universidad Autónoma de Barcelona, Barcelona.

Cha, M.; Haddadi, H.; Benevenuto, F.; Gummadi, Krishnap P. (2010): Measuring user influence in Twiter: The millon followers fallacy. En ICWSM, Proceedings of International AAAI Conference on Weblogs and Social. Disponible en: https:// goo.gl/88FwaW [Consultado el 10/06/2017].

Cadavid-Gómez, H. D. (2004): "Marketing de emociones. La forma para lograr fidelidad de los clientes", Semestre Económico, vol. 7, n. 13, pp. 203-211, Universidad de Medellín, Colombia. Disponible en: https://goo.gl/E2khKR [Consultado el 10/06/2017].

Castelló-Martínez, A. (2013): “La estrategia de medios sociales, el Inbound Marketing y la estrategia de contenidos: marketing de atracción 2.0". En I Congreso Internacional de Comunicación y Sociedad Digital (1-15), Logroño. Disponible en: https://goo.gl/2rvgMp

Congosto, M. L.; Deltell, L.; Claes, Florencia y Osteso, J. M. (2013): “Análisis de la audiencia social por medio de Twitter. Caso de estudio de los premios Goya 2013", Icono 14, vol. 11, n. 2, pp. 53-82. Disponible en: https://goo.gl/5EluGL. [Consultado el 10/06/2017]. DOI: 10.7199/ril4.v11i2.577.

Del Pino-Romero, C. (2009): "El entorno digital en la nueva era de los medios y la publicidad: la metamorfosis del consumidor”, Razón y Palabra, vol. 66, n. 14, pp. 1-17. Disponible en: https://goo.gl/uXLnq1 [Consultado el 05/06/2017].

Del Pino-Romero, C. y Galán-Fajardo, E. (2010): “Internet y los nuevos consumidores. El nuevo modelo publicitario”, Telos, n. 82, pp. 55-64. Disponible en: https://goo.gl/NSYuUt [Consultado el 12/04/2017].

Deza, M. (2012): Tu cerebro lo es todo, ¿sabes cómo y por qué decides? Madrid: Plataforma Editorial, 2012.

Echegaray-Eizaguirre, L. y Peñafiel-Saiz, C. (2013): "La utilización de las redes sociales como nuevas herramientas aplicadas al análisis de audiencia”, Trípodos, n. 33, pp. 157-172. Disponible en: https://goo.gl/mwKBDv [Consultado el $10 / 05 / 2017]$.

Felt, M. (2016): "Social media and the social sciences: How researchers employ Big Data analytics", Big Data \& Society. January-June:pp. 1-15. Disponible en: https://goo.gl/rXoYgu [Consultado el 11/03/2017].DOI: 10.1177/2053951716645828

Garay-Cruz, L. M. (2006): “iQué ha cambiado y qué sigue igual? Reflexiones sobre el concepto de audiencia mediática”, Revista Digital Universitaria, vol. 7, n. 6, pp. 1-10. Disponible en https://goo.gl/Rbs2pu [Consultado el 10/06/2017]. 
Godin, S. (1999): Permision Marketing: Turning Strangers into Friends, and Friends into Customers. New York: Simon\&Schuster.

Godin, S. (2001): El marketing de permiso. Barcelona: Granica.

Gil de Zúñiga, H.; Garcia-Perdomo, V.; McGregor, Sh. C. (2015): "What is second screening? Exploring motivations of second screen use and its effect on online political participation”, Journal of Communication, n. 65, pp. 793-815. Disponible en: https://goo.gl/6hbKBA [Consultado el 17/03/2017]. DOI: 10.1111/jcom.12174

GlobalwebIndex. (2017): The State of Mobile Ad-Blocking in 2017. Disponible en: https://goo.gl/PvXwRD [Consultado el 16/03/2017].

Groshek, J. y Krongard, S. (2016): "Netflix and Engage? Implications for Streaming Television on Political Participation during the 2016 US Presidential Campaign”, Social Science, vol. 5, n. 4, p.65. Disponible en: https://goo.gl/r8orR0 [Consultado el 15/04/2017]. DOI: 10.3390/socsci5040065

Gutiérrez, A. (2005): El jardín de las emociones musicales. Disponible en: https://goo.gl/opQGJ6 [Consultado el 17/03/2017].

Halligan, B. y Shah, Dh. (2009): Inbound Marketing: Get Found Using Google. Social Media and Blogs. New Jersey: John Wiley \& Sons.

Hallinan, B. y Striphas, T. (2016): "Recommended for you: The Netflix Prize and the production of algorithmic culture", New Media \& Society. vol 18, n.1, pp. 117-137. Disponible en: https://goo.gl/cMS25p [Consultado el 08/03/2017]. DOI: $10.1177 / 1461444814538646$.

Hornos, C. (2015): Influencers en la era digital (Tesis Doctoral). Universidad del Salvador.

Huertas, A. (2006): “Sobre la construcción ontológica del concepto de audiencia”, Fronteiras-Estudios Mediáticos, vol. VIII, n. 3, pp. 196-202. Disponible en: https://goo.gl/dXsLkz [Consultado el 10/02/2017].

Johnston, K. y McQuivey, J. L. (2017): The End of Advertising as We Know It. Disponible en: https://goo.gl/36fy9e [Consultado el 07/02/2017].

Lamas, C. (2010): "Los medios interactivos y su publicidad. La medición de audiencias". Telos, n. 82, pp. 95-101. Disponible en: https://goo.gl/fubWhP [Consultado el 10/03/2017].

Launchmetrics (2017): Informe sobre el estado del marketing de influencers. Disponible en: https://goo.gl/9v1Kuw [Consultado el 03/09/2017].

Lotz, A. (2007): The Television Will Be Revolutionized. New York: NYU Press.

Maldonado, S. (2015): Analítica web. Medir para triunfar. $3^{\circ}$ edición, Madrid: ESIC Editorial.

Marshall, D. (2004): New Media Cultures. Londres: Arnold Publishers.

Martín-Parreño, J. (2006): “Los contenidos publicitarios y el nuevo consumidor de medios digitales: del consumer al prosumer”. En II Congreso Online del Observatorio para la Cibersociedad. Disponible en: https://goo.gl/Rm9u8W [Consultado el 20/06/2017]. 
Martínez-Martínez, S. y Lara-Navarra, P. (2014): "El Big Data transforma la interpretación de los medios sociales”, $E l$ Profesional de la Información, vol. 23, n. 6, pp. 575-581. Disponible en: https://goo.gl/sgZt7R. https://doi.org/10.3145/ epi.2014.nov.03 [Consultado el 19/06/2017].

Mayer, V. (2016): "Production and Audience Studies: Together At Last The Places Where Audience Studies and Production Studies Meet”, Television \& New Media, vol. 17, n. 8, pp. 706-718. Disponible en https://goo.gl/WLxQD1 [Consultado el 10/03/2017]. DOI: 10.1177/1527476416652482.

Medinaveitia, E.; Merchante, M. (2015): “Medición de audiencias: desafío y complejidad en el entorno digital”, Harvard Deusto Marketing y Ventas, n. 131, pp. 26-33. Disponible en: https://goo.gl/rW5GLD [Consultado el 25/06/2017].

Meerman Scott, D. (2007): The New Rules of Marketing and PR. New Jersey: John Wiley \&Son, Inc.

Papí-Gálvez, N. y Escandell-Poveda, R. (2014): “El target group de la campaña: Definición, medición y tipología de selección online según el público", Claves en la planificación de la publicidad online: fundamentos, herramientas y retos. Madrid: AIMC, pp. 40-68.

Pintado-Blanco, T. y Sánchez-Herrera, J. (2014): Nuevas tendencias de comunicación estratégica, $3^{\mathrm{a}}$ ed. Madrid: ESIC.

Quintas-Froufe, N. y González-Neira, A. (2014): “Audiencias activas: participación de la audiencia social en la televisión”, Comunicar, vol. XXI, n. 43, pp.83-90. Disponible en https://goo.gl/oF77UM [Consultado el 14/07/2017]. DOI: 10.3916/ C43-2014-08.

Sanagustín, E. (2013): Marketing de contenidos: Estrategias para atraer clientes a tu empresa. Madrid: Anaya Multimedia. Manovich, L. (2017): Cultural Analytics, Social Computing and Digital Humanities, En Schäfer, M. T. y Es, K.V. The Datafied Society. Studying Culture through Data. Amsterdam: Amsterdam University Press. Disponible en: https://goo.gl/2v5Hyj [Consultado el 06/07/2017]. DOI: 10.5117/9789462981362.

Serrano-Puche, J. (2012): "Herramientas web para la medición de la influencia digital: análisis de Klout y Peerindex”, $E l$ profesional de la Información, vol. 21, n. 3, pp. 298-303. Disponible en: https://goo.gl/cD5LxY [Consultado el 04/05/2017]. DOI: 10.3145/epi.2012.may.11.

Serrano-Puche, J. (2017): "Metaanálisis del consumo digital en el ecosistema mediático contemporáneo: factores distintivos e implicaciones emocionales”, Revista Mediterránea de Comunicación/Mediterranean Journal of Communication, vol. 8, n. 1, pp. 75-85. Disponible en: https://goo.gl/TgUKbm. [Consultado el 11/03/2017]. DOI: 10.14198/MEDCOM2017.8.1.6. Steemeres, J. (2016): "International Sales of U.K. Television Content: Changeand Continuity in "the space in between" Production and Consumption”, Television \& New Media, vol. 17, n.8, pp. 734-753. Disponible en: https://goo.gl/NLEw3C [Consultado el 18/06/2017]. DOI: 10.1177/1527476416653481. 